\title{
AI Thinking for Cloud Education Platform with Personalized Learning
}

\author{
Paul Rad \\ University of Texas at San Antonio \\ Paul.rad@utsa.edu
}

\author{
Mehdi Roopaei \\ University of Texas at San Antonio \\ Mehdi.roopaei@utsa.edu
}

\author{
Nicole Beebe \\ University of Texas at San Antonio \\ Nicole.beebe@utsa.edu
}

\author{
Mehdi Shadaram \\ University of Texas at San Antonio \\ Mehdi.shadaram@utsa.edu
}

\author{
Yoris A. Au \\ University of Texas at San Antonio \\ Yoris.au@utsa.edu
}

\begin{abstract}
Artificial Intelligence (AI) thinking is a framework beyond procedural thinking and based on cognitive and adaptation to automatically learn deep and wide rules and semantics from experiments. This paper presents Cloud-eLab, an open and interactive cloudbased learning platform for AI Thinking, aiming to inspire i) Deep and Wide learning, ii) Cognitive and Adaptation learning concepts for education. It has been successfully used in various machine learning courses in practice, and has the expandability to support more AI modules. In this paper, we describe the block diagram of the proposed AI Thinking education platform, and provide two education application scenarios for unfolding Deep and Wide learning as well as Cognitive and Adaptation learning concepts. Cloud-eLab education platform will deliver personalized content for each student with flexibility to repeat the experiments at their own pace which allow the learner to be in control of the whole learning process.
\end{abstract}

\section{Introduction}

The history of Artificial Intelligence (AI) and computing can be traced back 5,000 years when the abacus was developed [1]. This instrument was developed based on number counting principles that informed the first computer programs and are still in use in parts of the world today. A multi-disciplinary scientific team began discussing the possibility of an artificial brain in 1940s and 1950s [2]. AI was founded as an academic discipline in 1956, the moment that AI obtained its name, its target, its first achievement and its key players [3]. In the 1980s an AI agenda called Expert Systems was introduced that simulated the knowledge and analytical skills of human experts. [4]. The discipline of AI, today more than a half a century old, has finally begun to accomplish some of its original goals, now that increasing computational power and more ready access thereto is possible. In the past two decades, we have seen enormously increased data availability (so called "Big Data"), more ready access to faster computers, and advanced machine learning techniques, all of which have been brought together to solve numerous challenges in cyberphysical-social [5]. Advances in deep learning have propelled forward research in image/video processing, text analysis, and voice recognition [6]. Deep learning includes techniques such as convolutional neural networks and recurrent neural networks and is a division of machine learning that leverages high level data abstractions and utilizes a deep graph with multiple processing layers [7-49].

AI as an educational tool has a thirty-year history [8]. It facilitates adaptive learning environments and other educational competencies that are flexible, customized, and effective [9]. AI used in an educational environment can illuminate the 'black box of learning' and can provide deep, wide, and a more fine-grained understanding of student learning [10].

In 2016, schools spent nearly $\$ 160$ billion on education technology and forecast spending to grow 17 percent annually through 2020 [45]. AI's share in the education market, specifically the learning process, will grow exponentially due to ability to provide personalized learning for each student and interpret complex human responses, such as emotions, while imparting knowledge on different subjects. Artificial intelligence will also play a key role in recruitment by matching the right person for the right position. A recent study estimated that by 2025 online talent platforms could enable as many as 60 million people find work that more closely suits their skills or preferences and reduce the cost of human resource management by as much as 7 percent [46]. Such benefits will compel private sector to invest in the research and development for intelligent education systems, which, in turn, will lead to the growth of this market.

\section{Related Works}

Jeannette Wing [11] introduced Computational Thinking as a "Universally applicable attitude and skill 
set everyone would be eager to learn and use". She opines that computational thinking should be taught to all children and regarded alongside reading, writing, and arithmetic in terms of its significance. In 2008, Wing proposed a further definition of computational thinking [12]. She argued for the cross-disciplinary applicability of computational thinking in research that everyone should taught. Her work became the clarion call bring computational thinking into all levels of education and application domains, including schools, universities, afterschool clubs, and etc. [13].

AI thinking and computational thinking posit that computing-enabled machine intelligence is a primary mechanism for realizing artificial intelligence [14]. And this is accomplished through the power of abstraction, the use of heuristics as a problem solving technique, and the importance of statistical learning. AI thinking is a problem solving platform based on cognitive thinking and deep and wide learning and it goes beyond computational thinking which mostly focuses on the logic and algorithm-based perspectives. [15].

In this paper a cloud-based AI-thinking platform, called Cloud-eLab, is proposed. The platform enables highly personalized learning based on the manual and perceptive feedback received from the students. The prepared computational contents can be tailored from the intermediate level to professional level and the platform can accept additional, new modules.

The rest of the paper is organized as follows. Section 3 describes definitions and fundamental elements of a computational thinking. Section 4 explains the theoretical background of AI-Thinking. role of cloud computing in educational systems. Section 5 presents the proposed AI-thinking platform in personalized learning and demonstrates two education modules i) a Mario game and, ii) facial emotion detection. Section 6 discusses future work and finally our conclusions are stated in section 7 .

\section{Computational Thinking: Definitions and Fundamental Elements}

Computational Thinking is interpreted as the solution of problems following the logical foundation used by computing professionals when they analyze and design systems [16]. There are several definitions introduced for the computational thinking concepts in the literature, described chronologically as follows:

- J. M. Wing in [11] proposed that "Computational thinking involves solving problems, designing systems, and understanding human behavior, by drawing on the concepts fundamental to computer science. Computational thinking includes a range of mental tools that reflect the breadth of the field of computer science".

- J.M. Wing and et al in [17] explained the definition of computational thinking as: "Computational thinking is the thought processes involved in formulating problems and their solutions so that the solutions are represented in a form that can be effectively carried out by an information-processing agent".

- The Royal Society of UK in [18] presented the computational thinking definition as: "Computational thinking is the process of recognizing aspects of computation in the world that surrounds us, and applying tools and techniques from Computer Science to understand and reason about both natural and artificial systems and processes".

- The Computer Science Teachers Association (CSTA) in [19] introduced a definition of computational thinking as: "computational thinking is a problem-solving methodology that expands the realm of computer science into all disciplines, providing a distinct means of analyzing and developing solutions to problems that can be solved computationally. With its focus on abstraction, automation, and analysis, CT is a core element of the broader discipline of computer science".

Clearly, the definition of computational thinking has evolved over time. Nevertheless, a common thread amongst them all is the focus on the systematic way of thinking and its use as a problem solving process. Another common thread is the absence of a data structure and method of finding patterns in a complicated data-driven problem. Consequently, while computational thinking is generally a well-defined framework for problem solving, it could be enhanced by integrating modern deep learning data analytics schemes. It could evolve further to a more advanced level of thinking and learning known as AI-thinking.

\section{AI-Thinking: Theoretical Background}

The evolution of theoretical approaches for cognitive science and knowledge representation is demonstrated in the Figure 1 in a chronological order; (i) Logic and Reasoning, (ii) Probability and Fuzzy, and (iii) Deep Data-Driven Learning which are the three major paradigms that have shaped the field of AI-thinking. The discipline of Artificial Intelligence has historical roots in logic. The logic-based model was concerned with reasoning and Automated Theorem Proving [47]. Robert C. Moore [48] argues three uses of logic in artificial intelligence; as a tool of analysis, as a basis for knowledge representation, and as a programming 
language. However, as the downfall of many knowledge-based reasoning models with large databases is that they cannot determine which data is relevant to the problem. Probabilistic methods came out of the need to deal with uncertainty in real world problem while the data driven AI-thinking provides cognitive learning model, using semantic analysis, perception, and context processing (mentioned in the Table 1), with more similarity to human thinking process rather than traditional thinking models.

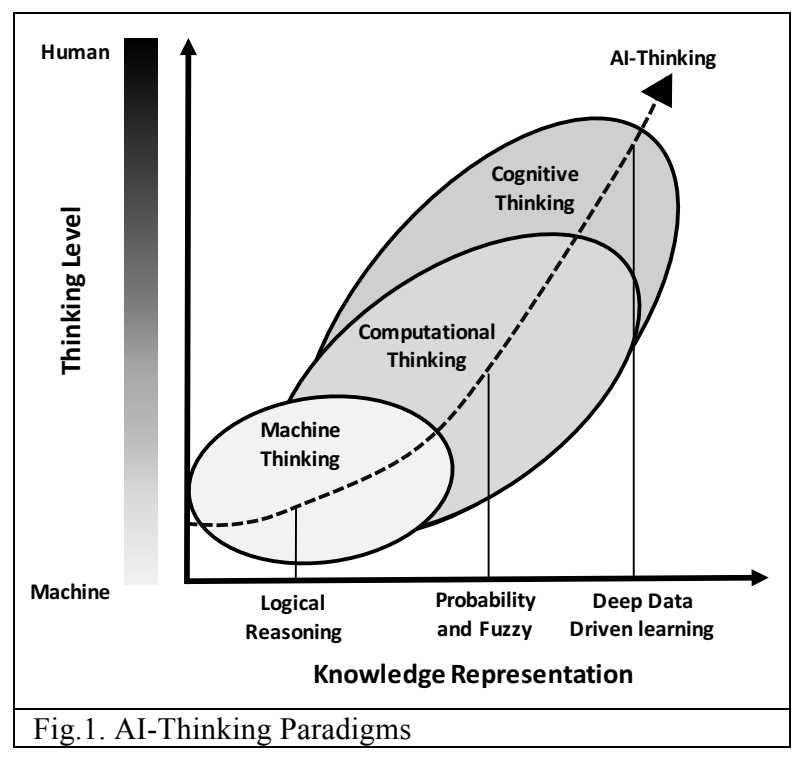

AI-thinking was first introduced in [20] as a learning process based on deep and wide learning and cognitive computing with the capability of semantic and/or context based analyzing of (un)structured data. In AIthinking, advanced data analytics methods such as machine learning and deep learning automatically decompose the problem by extracting complex features and there is no need for human cognitive to be in the loop to outline patterns. Therefore, the definition of AI-thinking could be:

AI-thinking is a framework that leverages data analytics with cognitive computing and adaptation to go beyond basic computational thinking and provide deep and wide learning by discovering patterns from experiments without explicitly knowing the constructs.

The model developed in this paper has utilized AIthinking to discover advanced cognitive and adaptation in educational environment and enhance students and instructors' communication by interpreting complex human responses while imparting knowledge on different subjects. The proposed thinking strategy goes beyond of computational thinking by employing deepwide learning and cognitive modules which distinguish the platform from the traditional educational-based framework: (i) deep and wide learning: the proposed AI-thinking platform provides educational attributes and features in highly scalable style where students could experiment variety of subjects based on their learning rate and interest. (ii) cognitive and adaptation: the proposed platform analyzes the educational environment and discover the influence of internal and external factors such as observation, categorization, and generalizations in order to produce personalized learning.

The proposed model developed in this article is an attempt to define an AI-thinking educational platform based on the theory depicted in Figure 2.

Hypothesis: The platform should provide enhanced educational-based connection between students and instructor by creating more understandable and easyto-use environment for students and afford the instructor to access more knowledge extracted by AI about the students' activities.

Variables: The main variables considered for the proposed model are: (i) Students' Learning and (ii) Educational Contents. The platform utilizes prescriptive model while imparting knowledge on different subjects to allow students understand contents in wide and deep levels.

Constructs: The proposed model constructs several sub-systems as follow: (i) adaptive feedback from student: This module captures and interprets complex student responses such as emotional intention to the contents, learning rate, and consequently adjusts educational variables and parameters. (ii) personalized learning: This module customizes content based on

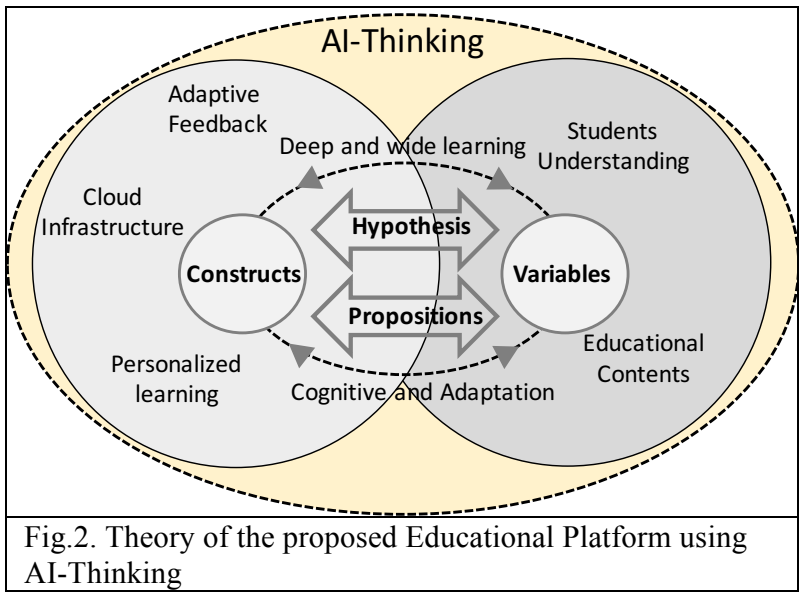

students learning and provides transparent visibility to instructors while protecting students' privacies (iii) cloud infrastructure: This model stores all education contents, student's feedback data logs, and processes batch and real-time analytics. 
Proposition: The proposed model measures students' Key Performance Indicator (KPI) while imparting knowledge on different subjects in their designed personalized settings. AI-thinking conducts not only predictive modeling but also prescriptive analytics for optimal educational content delivery or next step recommendations based on students' learning with instructor-in-loop to control and perhaps to adjust the situation accordingly.

The core concepts, skills and learning theorems for a traditional computational thinking and the proposed AI-thinking are addressed in Table. 1.

Table. 1. Core Concepts of Computational Thinking and the learning theories behind each element

\begin{tabular}{|c|c|c|}
\hline \multirow{2}{*}{ Core Concepts } & Definitions & Required Skill \\
\hline & \multicolumn{2}{|c|}{ Computational Thinking } \\
\hline \multirow[b]{2}{*}{$\begin{array}{l}\text { 1-Algorithmic } \\
\text { Thinking }\end{array}$} & $\begin{array}{l}\text { Algorithmic thinking is a way of getting to a } \\
\text { solution through a clear definition of the steps } \\
\text { [21]. }\end{array}$ & $\begin{array}{l}\text { The skill in algorithmic thinking is to set up } \\
\text { instructions or rules that if followed precisely } \\
\text { leads to answers to that and similar problems. }\end{array}$ \\
\hline & \multicolumn{2}{|c|}{$\begin{array}{l}\text { Plato theory proposed the question: How does an individual learn something new when the topic is } \\
\text { brand new to that person? This question may seem trivial; however, think of a human like a computer } \\
\text { The question would then become: How does a computer take in any factual information without } \\
\text { previous programming [24]? }\end{array}$} \\
\hline \multirow{2}{*}{ 2-Abstraction } & $\begin{array}{l}\text { Abstraction is the process of making an artefact } \\
\text { more understandable through reducing the } \\
\text { unnecessary detail [21]. }\end{array}$ & $\begin{array}{l}\text { The skill in abstraction is to choose the right } \\
\text { details to filter out, so that the problem becomes } \\
\text { easier, without losing anything that is important. }\end{array}$ \\
\hline & \multicolumn{2}{|c|}{$\begin{array}{l}\text { According to Cognitive Load theory, cognitive overload occurs when the required cognitive resources } \\
\text { to solve the problems exceeds cognitive resource availability. Cognitive overload is considered as a } \\
\text { major cause of learning failure [27]. }\end{array}$} \\
\hline \multirow[t]{2}{*}{ 3-Evaluation } & $\begin{array}{l}\text { Evaluation is the process of ensuring an } \\
\text { algorithmic solution is a good one- - that it fits the } \\
\text { purpose [22]. }\end{array}$ & $\begin{array}{l}\text { The skill in evaluation is to analyze, predict and } \\
\text { verify outcomes using test, trace, and logical } \\
\text { thinking. }\end{array}$ \\
\hline & \multicolumn{2}{|c|}{$\begin{array}{l}\text { Instructional design is the practice of creating instructional experiences that make the acquisition of } \\
\text { knowledge and skill more efficient, effective, and appealing [26]. }\end{array}$} \\
\hline \multirow[t]{2}{*}{ 4-Decomposition } & $\begin{array}{l}\text { Decomposition is a way of thinking about } \\
\text { problems, algorithms, artefacts, processes and } \\
\text { systems in terms of their components, [23]. }\end{array}$ & $\begin{array}{l}\text { The skill in decomposition is to find appropriate } \\
\text { components to make complex problems easier to } \\
\text { solve, novel situations better understood and } \\
\text { large systems easier to design. }\end{array}$ \\
\hline & \multicolumn{2}{|c|}{$\begin{array}{l}\text { A genetic decomposition describes the mental structures and mechanisms that a student might need to } \\
\text { construct in order to learn a specific mathematical concept [28]. }\end{array}$} \\
\hline \multirow{2}{*}{ 5-Generalization } & $\begin{array}{l}\text { Generalization is a way of quickly solving new } \\
\text { problems based on previous solutions to problems, } \\
\text { and building on prior experience [23]. }\end{array}$ & $\begin{array}{l}\text { The skill in generalization is associated with } \\
\text { identifying patterns, similarities and } \\
\text { connections, and exploiting those features to } \\
\text { solve a whole class of similar problems. }\end{array}$ \\
\hline & \multicolumn{2}{|c|}{$\begin{array}{l}\text { Gestalt theory, says that instead of obtaining knowledge from what's in front of us, we often learn by } \\
\text { making sense of the relationship between what's new and old. This theory proposes looking at the } \\
\text { patterns rather than isolated events. Gestalt views of learning have been incorporated into what have } \\
\text { come to be labeled cognitive theories [25]. }\end{array}$} \\
\hline+ & \multicolumn{2}{|c|}{ AI-Thinking } \\
\hline \multirow[t]{2}{*}{$\begin{array}{l}\text { 6-Deep and Wide } \\
\text { Learning }\end{array}$} & $\begin{array}{l}\text { Deep and wide learning is learning that takes root } \\
\text { in our apparatus of understanding, in the } \\
\text { embedded meanings that solve the problem using } \\
\text { previous and current experiences and knowledge } \\
\text { around it [29]. }\end{array}$ & $\begin{array}{l}\text { The skill in deep learning is the ability to model } \\
\text { high level abstractions, decomposition and } \\
\text { evaluation in data by using a deep graph with } \\
\text { many processing layers. }\end{array}$ \\
\hline & \multicolumn{2}{|c|}{$\begin{array}{l}\text { Deep and Surface learning is an approach and an attitude to learning, where the learner uses higher- } \\
\text { order cognitive skills such as the ability to analyze, synthesize, solve problems, and thinks meta- } \\
\text { cognitively in order to construct long-and-short term understanding [30]. }\end{array}$} \\
\hline \multirow{2}{*}{$\begin{array}{l}\text { 7- Cognitive and } \\
\text { Adaptation }\end{array}$} & $\begin{array}{l}\text { Cognitive and adaptation computing is the } \\
\text { simulation of human thought processes in a } \\
\text { computerized model with learning from } \\
\text { observations [31]. }\end{array}$ & $\begin{array}{l}\text { The skill in cognitive and adaptation computing is } \\
\text { to working with unstructured data using semantic } \\
\text { and context processing. }\end{array}$ \\
\hline & \multicolumn{2}{|c|}{$\begin{array}{l}\text { Cognitive Learning Theory is a broad theory that explains thinking and differing processes and how } \\
\text { they are influenced by internal and external factors such as observing, categorizing, and forming } \\
\text { generalizations about our environment in order to produce learning in individuals [32]. }\end{array}$} \\
\hline
\end{tabular}


AI-thinking provides a new philosophy of learning in order to orient not only scientific experiments but also challenges of everyday life. In recent years, computational thinking has proven to be a fundamental skill needed by everyone, however it would be more pertinent if it could discover and present hidden semantics and patterns for the learners, particularly in data driven experiments. Consequently, computational thinking should evolve to AI-thinking to provide deep and wide learning based on adaptive learning feedback of the learners. The educational society has been interested in designing appropriate teaching and pedagogical strategies by incorporating procedures for the cultivation and development of AI-thinking during the learning process.

\section{Proposed Personalized Cloud-Based AI- Thinking Platform}

In this article, we develop data analytics, cloud-driven AI-thinking platform, Cloud-eLab, that includes multiple Application Programming Interface (API)'s to encode various types of unstructured data (e.g., images, audio, text, and database data) and decompose them in semantic format and analyze the provided data using advanced machine learning techniques. The CloudeLab "www.cloudelab.org" proposed in this article is constructed based on the concept of an AI-thinking platform and provides the components required for any computational thinking structure. The platform components and structure are demonstrated in Fig. 3.

The contents created in this platform are computable, providing the learner the capability to easily change the environment (parameters and blocks) and repeat computable task as many times as needed. The contents are scalable and the students can learn, practice, and gradually make their knowledge deep step by step. The platform also has the potential to propose new related concepts automatically based on the capability and interest of the students for further learning concepts and modules. In fact, the platform makes a personalized learning environment where students can learn, practice, and run their own model. learning concepts and modules.

Cloud-eLab in the current format is designed for data analytics and network security learning, however, it could be extended to other learning domains with the easy addition of other new modules. The cloud environment enables individualized secure profiles, extensive data storage capacity, and team collaboration and knowledge and data sharing. Computation is handled in the cloud real-time, allowing access via low-end terminal platforms.

Cloud-eLab in the current format is designed for data analytics and network security learning, however, it could be extended to other learning domains with the easy addition of other new modules. The cloud environment enables individualized secure profiles, extensive data storage capacity, and team collaboration and knowledge and data sharing. Computation is handled in the cloud real-time, allowing access via low-end terminal platforms.

The block diagram of the proposed AI-Thinking educational platform is shown in Figure 3. Cloud-eLab includes of four main blocks: 1) Access control for providing secure connection to the platform and identifying users to load their educational profile. 2) Deep and wide learning for cognitive learning and educational adaptation is provided in AI-thinking block. 3) Computational thinking capability is integrated with the AI-thinking block to support any required level of thinking (learning) mentioned in Table 1. 4) Cloud computing supports collaboration, knowledge and data sharing, and access to massive storage and computational resources.

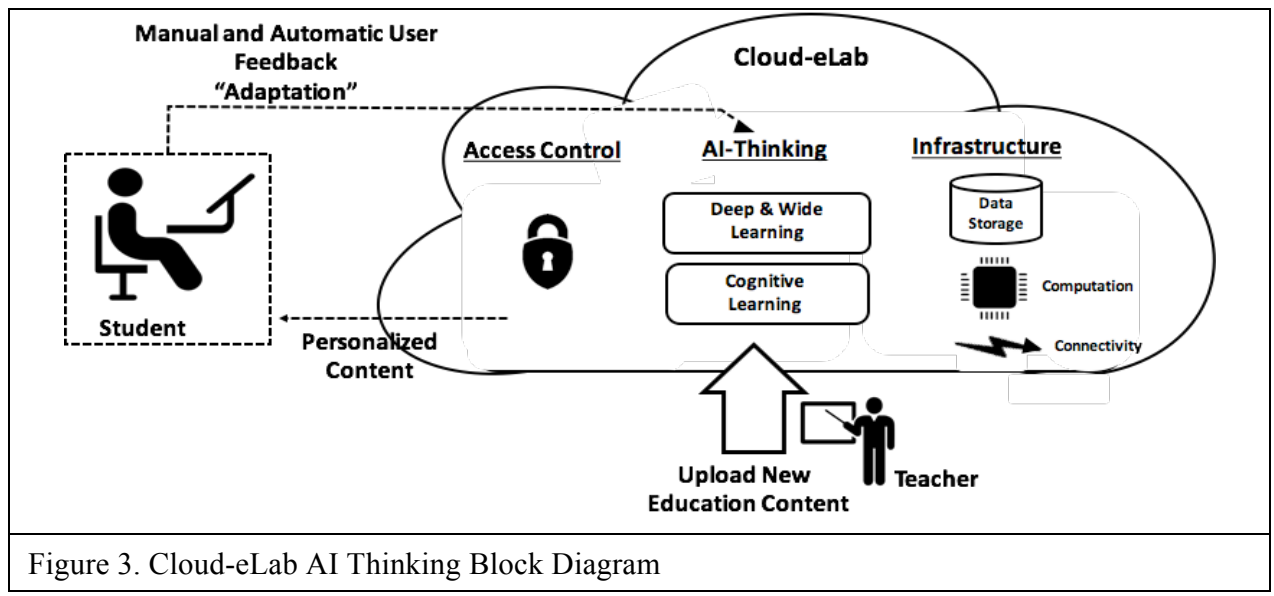


The Cloud-eLab is designed to deliver an educational AI thinking platform and constructed based on the following hypothesis: We hypothesize that the proposed system can provide a personalized learning environment, adaptable to individual student learning capability and domain expertise. This is accomplished through Cloud-eLab via the following four steps:

Step 1: Extracting relevant information from databases, quick prototyping with outstanding visualizations, rapid generation of diagrams, graphs, charts and other informational visualizations, and on-the-fly parameter variation. Effortless data ingestion from external databases in a diversity of formats, using high-level semantics to prepare data for instantaneous computation.

Step 2: Related data is examined and processed in the proposed cloud-based environment to develop a AI model of the problem, implemented as an API. Develop complex models using the world's largest web of algorithms, and position it as a restful API for easy programmatic access.

Step 3: The platform implements a desktop interface using any number of environments (e.g. IPython,
JavaScript, Jupyter Notebook), connecting it to the API model with custom bindings. Develop a front-end interface in any language or environment and easily connect it to the specific language code, built-in language interfaces or custom API bindings.

Step 4: Users can securely access the latest model at any time, manipulating parameters to simulate realworld scenarios. The platform provides fully configurable data security: User set-up and group policy application using standard authentication protocols, providing tight control over access to proprietary models. Use of pre-initialized API features and automatic load balancing to maximize the speed and availability of computations.

There are several data analytics modules has been developed by Cloud-eLab based on computational thinking such as: Natural Language Processing, Emotion Recognition based on Facial Expression and Voice, Face Detection, Network Security, Object Detection, Event Detection based on Social Media, and so on. A brief description of mentioned modules is expressed in the Table 3 .

Table 3. Developed computational thinking-based module in the proposed Cloud-eLab

\begin{tabular}{|l|l|l|}
\hline Module & Description & Applications \\
\hline $\begin{array}{l}\text { Natural } \\
\text { Panguage }\end{array}$ & $\begin{array}{l}\text { The domain of study that targets the interactions between human } \\
\text { language and computers, [36]. }\end{array}$ & $\begin{array}{l}\text { Automatic summarization, translation, } \\
\text { named entity recognition, relationship } \\
\text { extraction, sentiment analysis, speech } \\
\text { recognition, and topic segmentation. }\end{array}$ \\
\hline $\begin{array}{l}\text { Emotion } \\
\text { Recognition }\end{array}$ & $\begin{array}{l}\text { Emotion recognition is the method of recognizing human } \\
\text { emotion, most usually from facial expressions, [37]. }\end{array}$ & $\begin{array}{l}\text { Driver state surveillance, personalized } \\
\text { learning, health monitoring. }\end{array}$ \\
\hline $\begin{array}{l}\text { Network } \\
\text { Security }\end{array}$ & $\begin{array}{l}\text { Security in networks is the development of taking physical and } \\
\text { software protective measures to defend the essential networking } \\
\text { organization from unlicensed access, misuse, fault, modification, } \\
\text { improper data leakage or damage, thereby crafting a protected } \\
\text { platform for computers, users and programs to accomplish their } \\
\text { acceptable critical functions within a secure ecosystem, [38]. }\end{array}$ & $\begin{array}{l}\text { Security management include firewalls, } \\
\text { databases, Email, teleconferencing, } \\
\text { electronic commerce, intrusion } \\
\text { detection, and access control } \\
\text { applications. }\end{array}$ \\
\hline Face Detection & $\begin{array}{l}\text { Face detection is a computer technology being used in a variety } \\
\text { of applications that identifies human faces in digital images, [39] }\end{array}$ & $\begin{array}{l}\text { Face detection is used in biometrics, } \\
\text { video surveillance and any marketing } \\
\text { system that utilizing a webcam to detect } \\
\text { the race, gender, and age range of the } \\
\text { face. }\end{array}$ \\
\hline $\begin{array}{l}\text { Audio } \\
\text { Analytics }\end{array}$ & $\begin{array}{l}\text { Audio analytics is a method of learning the characteristics of } \\
\text { voice, speech, and sounds, [43-44]. }\end{array}$ & $\begin{array}{l}\text { Audio Analytics is used in music tag } \\
\text { retrieval system and voice recognition }\end{array}$ \\
\hline
\end{tabular}

Cloud-eLab Marketplace- The proposed AI-thinking platform, cloud-eLab, is a marketplace and education hub with the adoption of cloud computing capabilities to provide an opportunity to effectively change the field of computational science, and multimedia systems as researchers have access to mass distributed processing and data store resources. The platform has potential to provide a secure quantitative analysis cloud-driven AI-thinking platform with ease of use for online hands-on education and research solution exchange among educational communities. The marketplace was designed as a collaborative research platform providing workflow and tools to design, produce, and enhance multi-disciplinary collaborative research while educational Cloud-eLab attempts to make an online educational environment/content 
designed for mass quantitative analytics training and education.

The proposed AI-thinking platform has two additional components beyond the capability of computational thinking platforms: deep-wide learning and cognitive learning and adaptation. Here, two examples are considered to show how Cloud-eLab can enable solution development using AI-thinking. The first example involves the Mario game, which uses cognitive learning, and the second involves facial emotion detection, which is structured according to the deep and wide learning concept.

Cognitive learning and adaptation - Mario Game with Reinforcement Learning: Computer games have been used successfully in entry-level computer science courses and artificial intelligence classes to accelerate student interest and enthusiasm. Mario was selected because it can easily run the Cloud-eLab platform in a standard computer science lab, and because Mario has a robust visualizer that helps the students correct and understand their agent's rules. Further, it can be run in non-visualization mode, so that experiments can be run without having to wait for graphical calculations. The Mario project is build based on AI-Thinking particularly reinforcement learning on the Open-AI Gym [40].

Table. 4- Game learning based on AI-thinking

\begin{tabular}{|c|c|ccc|}
\hline \multirow{4}{*}{ Dimension 1 } & State & $x_{1}$ & $x_{2}$ & $x_{3}$ \\
\cline { 2 - 4 } & Act & Move-Left & Not-Move & Move-Right \\
\cline { 2 - 4 } & Reward & -1 & 0 & 1 \\
\hline Dimension 2 & State & $x_{4}$ & $x_{5}$ \\
\cline { 2 - 4 } & Act & Not-Jumping & Jumping \\
\cline { 2 - 4 } & Reward & 0 & 1 \\
\hline \multirow{2}{*}{\begin{tabular}{c} 
Dimension 3 \\
\cline { 2 - 4 }
\end{tabular}} & State & $x_{6}$ & $x_{7}$ \\
\cline { 2 - 4 } & Act & Running & Walking \\
\cline { 2 - 4 } & Reward & 0 & 1 \\
\hline
\end{tabular}

The reinforcement strategy for the total 12 distinct action is explained in Table 4. The agent's target in this example is to learn a strategy to maximize award acquisition. In this example, we focused on learning an action-reward function, which precisely predicts the long-term reward for an action in a given state. The whole procedure is based on AI- thinking that enables students to think at varied cognitive levels, decompose the problem into different actions, and develop a reward maximization strategy using AI-thinking, such as the ruleset identified in Table 4. Students can visually watch step-by-step learning and adaptively change their learning strategy based on feedback of agents in the game. In Figure 4, one of the cognitive concepts is demonstrated using the Mario size increase caused by the action of touching a mushroom. Students can change the options and parameters and design different actions based on their cognitive learning and adaptation.

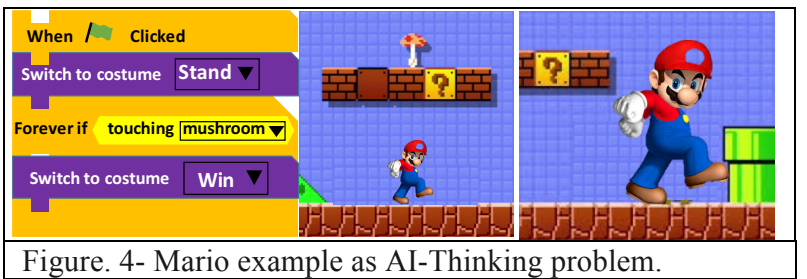

Deep-Wide Learning - Facial Emotion Detection: In this second example application of AI-thinking based education, we use the problem of facial emotion detection to demonstrate deep-wide learning. Basic human emotions translate to a variety of facial muscle movements. It is often easy for humans to read basic emotions such as happiness, sadness etc. from facial expressions. Teaching a neural network to classify between these basic emotions to match human level accuracy and beyond is a tedious task. The model should not only detect different sized faces, but also accurately generate emotion probabilities for the face.

Mathematically, the temporal deep learning model attempts to solve an optimization problem on a facial expression image database to find the optimal model over the selected training set to detect basic emotions. The model consists of several convolutional neural network layers with a very large number of learnable parameters between the layers to extract various action unit features in the facial images and discover the hidden patterns in them. Action units (AUs) are the major actions of individual muscles or sets of facial muscles. They are classified as additive or non-additive AUs according to whether they occur in combination or not.

The goal of the provided module in the proposed Cloud-eLab is to detect the facial emotion recognition based on computational thinking learning in image and videos. To recognize any emotion in image, the platform first abstracts the problem by detecting faces in the image and remove other unnecessary objects. Then the recognized face is decomposed to more sections and details. Then an advanced machine learning algorithm underlying the platform attempts to find patterns which could be matched with the existing features in a face such as eyes, lip, noise, ear, hair, and eyebrows. Pattern recognition and matching are executed on the heavy computational nodes on exiting huge pre-trained models. Finally, the probability of basic emotions existed in detected faces are computed and the face is labeled based on the maximum value of identified emotion. The platform is designed to deliver optimal architecture for any specific problem according to the size of database, the desired pre- 
defined accuracy, and near real-time processing. Cloud-eLab then has the ability to generalize the mood of an input facial image, such as the detection of happy in Figure 2 below. In this example, the deep-wide strategy automatically extracts the required emotion patterns in a face by applying filters intelligently on the image.

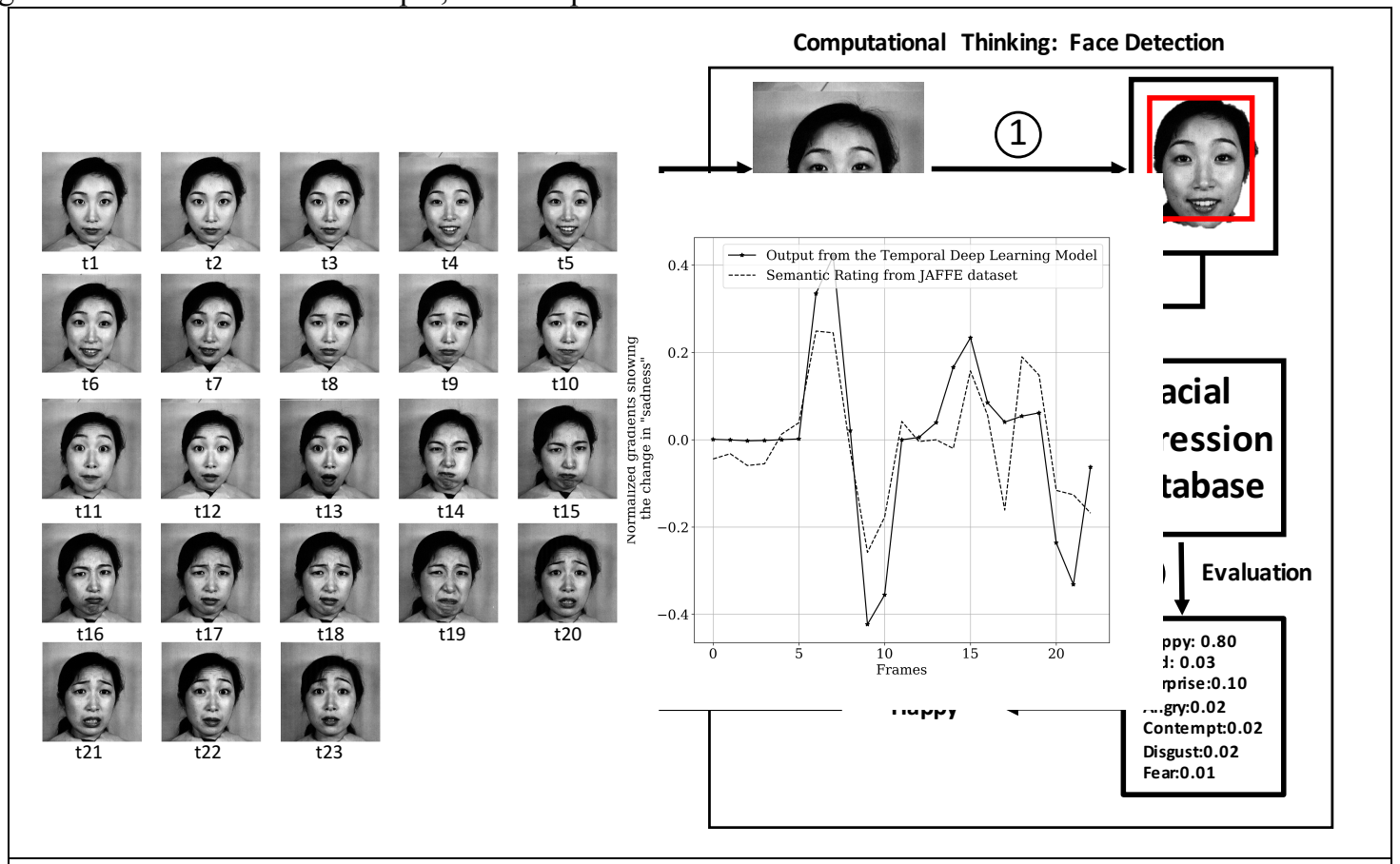

Figure 5. Facial Emotion Detection based on Facial Expression proposed at Cloud-eLab with representation in the form of AI-Thinking-(Images sourced from [41])

Platform Evaluation in Educational Setting- The platform was implemented in some classes in the University of Texas at San Antonio (UTSA), within the Electrical and Computer Engineering department, in 2016-2017 academic calendar. The Cloud-eLab mostly employed for graduate level classes related to data analytics. To provide a testbed for the proposed AI thinking platform several components are designed and constructed as: (i) required computational node and data storage was delivered on the Chamaeleon Cloud which is the first academic cloud environment granted by National Science Foundation [42], (ii) content of the course is provided as computable document format developed by Open Cloud Institute (OCI) at UTSA , and; (iii) The programming language utilized for this platform is a simple, unique with strong visualize capabilities at the different level and scale of problem solving designed by OCI.

The platform is highly exploited for courses which mostly focused on hands on projects where students could understand all the concepts step by step in their defined projects. AI thinking let the students to design an architecture based on a specific data analytics problem, however the students were then instructed to modify or re-design the supplied architecture to find the solution with reasonable accuracy. All the interface libraries were preinstalled on the cloud environment and other requested learning modules requested by the students were added to the platform.

\section{Future Work}

The platform needs the enhanced capability to: 1) intelligently understand and recognize student struggle and ambiguities that might occur during the learning process, and 2) offer a lemma or supportive documentation to assist the student resolve their learning challenge. Additionally, the platform could be expanded to incorporate new learning technologies, such as augmented and/or virtual reality. The proposed platform should provide high-level cognitive development to analyze and might also identify if and when a student is confused, bored, or unfulfilled, to help teachers recognize and improve a learner's emotional readiness for learning while preserving all the standard privacy issues. In fact, the platform will not support only AI-thinking but system thinking would be also provided to understand Cloud-eLab platform by examining the linkages and interactions between the components that comprise the entirety of that defined system. 


\section{Conclusion}

This paper presents an educational, cloud-driven platform called Cloud-eLab that enables AI-thinking based learning and problem solving. The platform provides personalized learning based on automatic cognitive feedback from the students and offer contents to increase the learning rate and enhance their interest. It supports scalable computable content and extendable modules at all educational levels. The platform goes beyond computational thinking and incorporates the additional components of the AI-thinking philosophy to present the problem in deep and wide manner and providing cognitive learning and adaptation. Thereby, the proposed system provides an abstraction mode by hiding unnecessary portions and decomposing the problem into multi sub-problems, which helps students understand complex patterns more easily. The proposed Cloud-eLab has the capability to: (i) deal with open ended problems, (ii) represent ideas in a computationally meaningful way, (iii) break down large problems into smaller ones, (iv) evaluate strengths and/or weaknesses of problem representation, and (v) generate algorithmic solutions. Cloud-eLab was applied and evaluated in a graduate machine learning course over two semesters.

\section{Acknowledgement}

We gratefully acknowledge the following:(i) Support by NSF grant CNS-1419165 to the University of Texas at San Antonio; and (ii) time grants to access JetStream Cloud and Chameleon Cloud (NSF Cloud). The authors would like to thank Mr. Gregory Hooks, Senior Software developer at Open Cloud Institute, for his valuable effort for implementing the platform.

\section{References}

[1] Spyros Makridakis, The Forthcoming Artificial Intelligence (AI) Revolution: Its Impact on Society and Firms, Neapolis University of PAPHOS, Working papers Series, 2017/01.

[2] Mc Corduck, Pamela, 2004. Machines Who Think (2nd ed.), Natick, MA: A. K. Peters, Ltd., ISBN 1-56881-205-1, OCLC 52197627.

[3] Russell, Stuart J.; Norvig, Peter, 2003. Artificial Intelligence: A Modern Approach (2nd ed.), Upper Saddle River, New Jersey: Prentice Hall, ISBN 0-13-790395-2.

[4] Crevier, Daniel, 1993. AI: The Tumultuous Search for Artificial Intelligence, New York, NY: BasicBooks, ISBN 0465-02997-3.

[5] Kurzweil, Ray (2005), The Singularity is Near, Viking Press, ISBN 0-14-303788-9, OCLC 71826177.
[6] Markoff, John (14 October 2005), "Behind Artificial Intelligence, a Squadron of Bright Real People", The New York Times, retrieved 16 October 2008.

[7] Rohith Polishetti, Mehdi Roopaei, Paul Rad, A NextGeneration Secure Cloud-Based Deep Learning License Plate Recognition for Smart Cities, 15th IEEE International Conference on machine Learning and Application, Anaheim California, pp. 286-293, 2016.

[8] Luckin, R., Holmes, W., Griffiths, M. \& Forcier,L. B. (2016). Intelligence Unleashed. An argument for AI in Education.London: Pearson.

[9] Todd W. Neller, 2017. AI Education: Open-Access Educational Resources on AI, AI Matters, Vol. 3, Issue 1. DOI: $10.1145 / 3054837.3054841$.

[10] Hill, P. \& Barber, M. (2014). Preparing for a renaissance in assessment. London: Pearson., DiCerbo, K. E. \& Behrens, J. T. (2014). Impacts of the digital ocean on education. London: Pearson.

[11] Wing, J.M., 2006. Computational thinking. Communications of the ACM, 49(3), pp.33-35.

[12] Wing, J.M., 2008. Computational thinking and thinking about computing. Philosophical transactions of the royal society of London A: mathematical, physical and engineering sciences, 366(1881), pp.3717-3725.

[13] NRC (2012). A framework for K-12 science education: Practices, crosscutting concepts, and core ideas. The National Academies Press.

[14] George Gadanidis, 2017 Artificial intelligence, computational thinking, and mathematics education, The International Journal of Information and Learning Technology, Vol. 34 Issue: 2, pp.133-139, https://doi.org/10.1108/IJILT-09-2016-0048

[15] Piyanuch Silapachote, Ananta Srisuphab, 2016. Teaching and learning computational thinking through solving problems in Artificial Intelligence: On designing introductory engineering and computing courses, IEEE International Conference on teaching, Assessment, and Learning for Engineering (TALE), http://10.1109/TALE.2016.7851769

[16] Weintrop, D. et al., 2016. Defining computational thinking for mathematics and science classrooms. Journal of Science Education and Technology, vol. 25, pp. 127-147.

[17] Wing, J. M. (2011). Research Notebook: Computational Thinking-What and Why? The Link.

[18] The Royal Society. (2012). Shut down or restart? The way forward for computing in UK schools.

[19] CSTA Standards Task Force. (2016). [Interim] CSTA K-12 Computer Science Standards. New York: CSTA. 
[20] Daniel Zeng, 2013. From Computational Thinking to AI Thinking, IEEE Intelligent System, Letter to the Editor, PP. 2-4, DOI: 10.1109/MIS.2013.141.

[21] Csizmadia, A., Curzon, P., Dorling, M., Humphreys, S., Ng, T., Selby, C., \& Woollard, J. (2015). Computational thinking A guide for teachers. Computing at School.

[22] Lee, I., Martin, F., Denner, J., Coulter, B., Allan, W., Erickson, J., ... Werner, L. (2011). Computational thinking for youth in practice. ACM Inroads, vol. 2(1), pp. 32-37.

[23] Sze Yee Lye, Joyce Hwee Ling Koh. (2014). Review on teaching and learning of computational thinking through programming: What is next for $\mathrm{K}-12$ ?. Computers in Human Behavior, vol. 41, pp. 51-61.

[24] Lee, Myungjoon, "Plato's philosophy of education: Its implication for current education" (1994). Dissertations (1962 - 2010) Access via Proquest Digital Dissertations. AAI9517932.

[25] Ellis, W.D. (1938). A Source Book of Gestalt Psychology. New York: Harcourt, Brace \& World.

[26] Dick, W. (1991). An instructional designer's view of constructivism. Educational Technology, May, 41-44.

[27] Sweller, J., Cognitive load during problem solving: Effects on learning, Cognitive Science,Vol. 12, 1988, pp. 257-285.

[28] Ilana Arnon, Jim Cottrill Ed Dubinsky Asuman Oktaç · Solange Roa Fuentes María Trigueros · Kirk Weller, APOS TheoryA Framework for Research and Curriculum Development in Mathematics Education, Springer, 2013.

[29] Heng-Tze Cheng et al., 2016, Wide \& Deep Learning for Recommender Systems, arXiv:1606.07792.

[30] Stanger-Hall, K. F., 2012. Multiple-choice exams: An obstacle for higher-level thinking in introductory science classes. Cell Biology Education-Life Sciences Education, vol. 11 (3), 294-306.

[31] John E. Kelly III, 2015. Computing, cognition and the future of knowing How humans and machines are forging a new age of understanding, IBM Global Services.

[32] Evans, M. A., and Carr, T. H., 1985. Cognitive abilities, conditions of learning, and the early development of reading skill. Reading Research Quarterly, vol. 20, 327-50.

[33] Mehdi Roopaei, Paul Rad, Mo Jamshidi, Deep Learning Control for Complex and Large Scale Cloud Systems, Intelligent Automation and Soft Computing (AUTOSOFT), DOI: 10.1080/10798587.2017.1329245, 2017.

[34] Samar I. Swaid., 2015. Bringing computational thinking to STEM education, Procedia Manufacturing, vol. 3, pp. $3657-3662$.
[35] Jir Ikopecky, (2014). Using computable document format in the teaching mathematics. 2014: Proceedings TIME, ISSN 2313-1640.

[36] Goldberg, Yoav, 2016. A Primer on Neural Network Models for Natural Language Processing. Journal of Artificial Intelligence Research vol. 57, pp. 345-420.

[37] Miyakoshi, Yoshihiro, and Shohei Kato. (2011). Facial Emotion Detection Considering Partial Occlusion Of Face Using Baysian Network". Computers and Informatics, pp. 96-101.

[38] SA Singh, P Najafirad, , P Najafirad, 2009. Systems and methods for accessing a shared storage network using multiple system nodes configured as server nodes, US Patent $7,506,009$.

[39] A Kwaśniewska, J Rumiński, P Rad, (2017). Deep features class activation map for thermal face detection and tracking. 10th International Conference on Human System Interactions (HSI), pp. 41-47.

[40] Greg Brockman et al., 2016. Open-AI Gym, arXiv:1606.01540.

[41] Lyons Michael J., Akemastu Shigeru, Kamachi Miyuki, and Gyoba Jiro. (1998). Coding Facial Expressions with Gabor Wavelets, 3rd IEEE International Conference on Automatic Face and Gesture Recognition, pp. 200-205.

[42] Chameleon Cloud: A configurable experimental environment for large-scale cloud research. Chameleoncloud.org. $\quad$ Retrieved, from https://www.chameleoncloud.org/. 2017.

[43] Sharaj Panwar, Arun Das, Mehdi Roopaei, Paul Rad, A Deep Learning Approach for Mapping Music Genres, 12th Annual System of System Engineering Conference, June 1821 2017, Hawaii, USA.

[44] Andrew Boles, Paul Rad, Voice Biometrics: Deep Learning-based Voiceprint Authentication System, $12^{\text {th }}$ Annual System of System Engineering Conference, June 1821 2017, Hawaii, USA

[45] 2016. Global EdTech industry report: A map for the future of education," EdTechXGlobal and Ibis Capital.

[46] 2015. A labor market that works: Connecting talent with opportunity in the digital age, McKinsey Global Institute.

[47] Loveland, Donald W, 2016. Automated Theorem Proving: a logical basis. Elsevier.

[48] Moore, Robert C. 1995, Logic and representation. Vol. 39. Center for the Study of Language (CSLI).

[49] N Gallardo, N Gamez, P Rad, M Jamshidi, (2017). Autonomous decision making for a driver-less car. System of Systems Engineering Conference (SoSE), pp. 1-6. 